\title{
A NEW TYPE OF CLASS NUMBER RELATIONS*
}

BY

E. T. BELL

1. Beyond the well known remark that the tenth of Kronecker's class number relations is equivalent to the theorem of Gauss which gives the number of representations of an integer as a sum of three squares, the class number relations implicit in the representations of integers as sums of an odd number of squares have not been developed. The remaining relations obtainable from this source are of types different from any in the literature, and are only remotely connected with elliptic functions. They are of two kinds: relations involving in addition to the class number functions either (1) only functions of the real divisors of integers, or (2) functions of the complex divisors of integers or of the representations of integers in quadratic forms containing more than three indeterminates, e. g., $\sum a b c d$, the $\sum$ extending to all integers $a, b, c, d$ the sum of whose squares is a constant. The second kind is of but slight interest, as there obviously is no difficulty in obtaining any number of such relations. But those of the first kind are much less common and in fact appear to be finite in number, being furnished by representations as sums of $5,7,9,11$, 13 or 15 squares, but not by representations as sums of 17, 19, 21, 23 or 25 squares, and probably by no higher odd number of squares.

The relations of the first kind fall into two distinct species. The first of these comprises equalities, for different $q, r, s, t, u, K$, between two or more sums of the form

$$
\sum_{a} f(q n+r-s a) K(t a-u)
$$

where $n, q, r, s, t, u$ are constant positive integers, $f(n)$ is a function of the real divisors alone of $n$ for all integers $n, K$ is one of $E, F, F_{1}, G$, and the summation extends to all $a=1,2,3, \ldots$ that make both $q n+r-s a$ and $t a-u \geqq 0$. In a paper not yet published I have made a complete determination of this species.

The second species, in many ways more interesting than the first, is as follows. In general when $n$ is unrestricted no sum of type $(A)$ in a relation of the first species is reducible to a function of only the real divisors of a single integer. But when $n$ is of the form $c n_{1}^{2}$, where $c$ is a constant

\footnotetext{
* Presented to the Society, San Francisco section, October $25,1924$.
} 
integer, the sums are in general so reducible. A relation of the second species is then one in which a sum of type $(A)$ with $n$ replaced by $c n_{1}^{2}$ is equal to a function of the real divisors only of a single integer.

The present paper is limited to a short summary in $\S 2$ of the method for finding relations of the second species, to the presentation in $\S 4$ of the seven simplest specimens, and to the proofs of these in $\S 5$. The entire set of relations of the second species can be derived by applying the method as outlined to the formulas of the paper already cited (to appear elsewhere) in conjunction with the results of the paper quoted in $§ 2$.

2. Let $N_{r}(n)$ denote the number of representations of $n$ as a sum of $r$ squares with integer roots $\gtreqless 0$, and $N_{r}(n, s)$ the number of representations of $n$ as a sum of $r$ integer squares precisely $s$ of which are odd with roots $\gtrless 0$ and $r-s$ even with roots $引 0$. Then $N_{r}(4 n, 0)=N_{r}(n)$, $N_{r}(0,0)=1$, and by considering in all possible ways a sum of $r$ squares as being composed of a sum of $r-3$ squares plus a sum of 3 squares, it is easily seen that

$$
\begin{aligned}
N_{r}(n-1, s) & =\frac{4 r(r-1)(r-2)}{s(r-s)(r-s-1)} \sum F(4 a-3) N_{r-3}(n+2-4 a, s-1), \\
N_{r}(n-1, s) & =\frac{4 r(r-1)(r-2)}{s(s-1)(r-s)} \sum F(4 a-2) N_{r-3}(n+1-4 a, s-2), \\
N_{r}(n-1, s) & =\frac{8 r(r-1)(r-2)}{s(s-1)(s-2)} \sum F(8 a-5) N_{r-3}(n+4-8 a, s-3), \\
N_{r}(n-1, r-3) & =2 r(r-1)(r-2) \sum E(a-1) \quad N_{r-3}(n+3-4 a, r-3), \\
N_{r}(n) & =12 E(n)+12 \sum_{a=1}^{n} E(a-1) N_{r-3}(n+1-a),
\end{aligned}
$$

in which, as always henceforth, the $\Sigma$ without indicated limits refers to all $a=1,2,3, \ldots$ that make the arguments of the summands $\geqq 0$. As usual $F(n)$ denotes the number of odd classes of binary quadratic forms of determinant $-n, E(n)=F(n)-F_{1}(n)$, where $F_{1}(n)$ is the number of even classes, and $G(n)$ is the whole number of classes. The general formula of which the above five are special cases is discussed in the paper mentioned in $\S 1$. There is no difficulty in proving the five independently from the well known theorems $(n=1,2,3, \ldots)$

$$
\begin{aligned}
& N_{3}(8 n-5)=N_{3}(8 n-5,3)=8 F(8 n-5), \\
& N_{3}(4 n-3)=N_{3}(4 n-3,1)=12 F(4 n-3), \\
& N_{3}(4 n-2)=N_{3}(4 n-2,2)=12 F(4 n-2), \\
& N_{3}(n-1)=12 E(n-1) .
\end{aligned}
$$


For $r=3,5,7,9,11,13,15$ these formulas give the relations of the first species in $\S 1$.

There is a corresponding set of formulas, not involving the class number functions, obtained from the consideration of a sum of $r$ squares as a sum of $r-1$ squares plus a single square in all possible ways. The results derived from this set are given in an earlier paper*.

When the same $N_{r}(n)$ or $N_{r}(n, s)$ is listed by both methods we equate the results and obtain the initial formula from which is derived a relation of the second species ( $\S 1$ ). The procedure then is as follows. One member of this initial formula is (loc. cit., p. 170) a sum of the form $\sum f\left[\left(p n-q a^{2}\right) / g\right]$, in which $p, q, g$ are numerical constants, or it is the sum of a small number (not more than 4) such sums. If now it is possible to choose a constant $c$ such that each sum of this form reduces, when $n$ is replaced by $c n^{2}$, to the form $\sum f\left[\gamma\left(\alpha^{2} n^{2}-\beta^{2} a^{2}\right)\right]$, we can apply the method of Hurwitzt and express each sum as a function of the real divisors alone of $n$. This process can be applied to the numerous formulas obtained as outlined above from $N_{r}(n), N_{r}(n, s)$ for $r=3,5,7,9,11,13$, and this apparently exhausts its scope.

3. To state the simplest examples of the second species we shall require $\xi(n)$ (the number of divisors of $n$ of the form $4 k-3$ minus the number of divisors of the form $4 k-1$ ); $\zeta_{r}(n)$ (the sum of the $r$ th powers of all the divisors of $n$ ). As always henceforth $n=2^{\alpha} m, \alpha \geqq 0, m$ is odd and $m=\prod p^{\beta} \equiv p^{\beta} q^{\gamma} \ldots$ is the resolution of $m$ (when $m>1$ ) into powers of distinct primes $p, q, \cdots$.

The theorems of Stieltjes and Hurwitz $\ddagger$ can be restated in the form

$$
N_{3}\left(n^{2}\right)=6 S(m), \quad N_{3}\left(n^{2}\right)=10 \zeta_{3}\left(2^{\alpha}\right) H(m)
$$

in which

$$
\begin{aligned}
& S(m)=\prod\left[p^{\beta}+\left\{1-(-1)^{(p-1) / 2}\right\} \zeta_{1}\left(p^{\beta-1}\right)\right] \\
& H(m)=\prod\left[\zeta_{3}\left(p^{\beta}\right)-p \zeta_{3}\left(p^{\beta-1}\right)\right]
\end{aligned}
$$

the product extending to $p^{\beta}, q^{\gamma}, \ldots ; S(1)=H(1)=1$.

* American Journal of Mathematics, vol. 42 (1920), pp. 168-188.

†Comptes Rendus, vol. 98 (1884), pp. 504-507.

$\ddagger$ Stieltjes, Comptes Rendus, vol. 98 (1884), pp. 663-4; Hurwitz, loc. cit. It does not seem to have been noticed that Hurwitz' theorem on $N_{3}\left(n^{2}\right)$, L'Intermédiaire des Mathématiciens, vol. 14 (1907), p. 107, is equivalent to Stieltjes' result of 1884; cf. Dickson, History of the Theory of Numbers, vol. 2, pp. ix, 271, vol. 3, pp. 134-5. 
For reductions of formulas we have

and

$$
\xi(4 k-1)=0, \xi(n)=\xi(m), \zeta_{3}\left(2^{\alpha+1}\right)=8 \zeta_{3}\left(2^{\alpha}\right)+1,
$$

$$
\begin{gathered}
E(8 n-5)=\frac{2}{3} F(8 n-5), E(8 n-1)=0, E(4 n)=E(n), \\
E(4 n-3)=F(4 n-3), E(4 n-2)=F(4 n-2), F(4 n)=2 F(n),
\end{gathered}
$$

to all of which we shall refer as the elementary reductions. Applying these to Stieltjes' theorem, and recalling that $N_{3}(n)=12 E(n)$, we get

$$
\begin{array}{ll}
F\left(n^{2}\right)=2^{\alpha-1} S(m), & F_{1}\left(n^{2}\right)=\frac{1}{2}\left(2^{\alpha}-1\right) S(m), \\
G\left(n^{2}\right)=\frac{1}{2}\left(2^{\alpha+1}-1\right) S(m), \quad E\left(n^{2}\right)=\frac{1}{2} S(m),
\end{array}
$$

for the last of which we shall have particular use later.

From now on all formulas have been checked numerically.*

By the usual conventions a class equivalent to $a\left(x^{2}+y^{2}\right)$ is counted $\frac{1}{2}$ in $F$ or $F_{1}$; one equivalent to $a\left(x^{2}+x y+y^{2}\right)$ counts for $\frac{1}{3}$ in $F_{1}$, and $F(0)=0, E(0)=G(0)=\frac{1}{12}$.

4. The seven simplest relations of the second species are

(I) $168 \sum \xi(a) E\left(n^{2}-a\right)$

$$
8 \sum \xi(a) F\left(m^{2}-4 a\right)
$$$$
=5\left(8^{\alpha+1}-1\right) H(m)-21 S(m) \text {, }
$$

$$
\sum \xi(8 a-7) F\left(4 m^{2}+7-8 a\right)=H(m),
$$$$
\sum \xi(8 a-3) F\left(16 n^{2}+3-8 a\right)=8^{\alpha+1} H(m) \text {, }
$$$$
\sum \xi(4 a-3) F\left(4 m^{2}+6-8 a\right)=H(m) \text {, }
$$

(VII) $12 \sum \xi(4 a-3) E\left(\frac{m^{2}+3}{4}-a\right)=H(m)$,

in all of which, by the notation explained, $n=2^{\alpha} m, \alpha \geqq 0, m$ odd. Let us first note how these are related among themselves by means of the elementary reductions. Evidently the left of (I) can be written

$168 \sum\left[\xi(a) E\left(n^{2}-4 a\right)+\xi(4 a-3)\left\{E\left(n^{2}+6-8 a\right)+E\left(n^{2}+3-4 a\right)\right\}\right]$.

* There is a table for $F(n), n=1$ to 100 , in the Tôhoku Mathematical Journal, vol. 9 (1921), p. 116. 
Hence it follows readily that (VII) is implied by (I) with $\alpha=0$ and (II); (III) is implied by (I) with $\alpha=1$ and (V); (IV) implied by (I) with $\alpha=2$ and (VI).

5. Although it is sufficient to prove (I), (II), (V), (VI), it is as quick to prove (I)-(VI) together. From the formulas in $\S 2$ with $r=5$ we get the following, which can be easily verified:

(1) $N_{5}(n)=12 E(n)+48 \sum E(a-1) \xi(n+1-a)$,

(2) $N_{5}(8 n-7)=20 F(8 n-7)+80 \sum F(4 a-3) \xi(2 n-1-a)$,

(3) $N_{5}(4 n)=12 E(n)+16 \sum[3 E(a-1) \xi(n+1-a)$ $+5 F(8 a-5) \xi(4 n+5-8 a)]$,

(4) $N_{5}(8 n-4)=12 E(2 n-1)+16 \sum[3 E(a-1) \xi(2 n-a)$ $+5 F(8 a-6) \xi(4 n+1-4 a)]$,

(5) $N_{5}(8 n)=12 E(2 n)+16 \sum[3 E(a-1) \xi(2 n+1-a)$ $+5 F(8 a-2) \xi(4 n+1-4 a)]$.

In (1) replace $n$ by $n^{2}$, in (2) $n$ by $\frac{1}{8}\left(m^{2}+7\right)$, in (3) $n$ by $n^{2}$, in (4) $n$ by $\frac{1}{2}\left(m^{2}+1\right)$, in (5) $n$ by $2 n^{2}$, and to the results thus obtained apply the theorems of Stieltjes and Hurwitz. By obvious combinations of the resulting formulas we get

$$
\begin{aligned}
24 \sum E(a-1) \xi\left(n^{2}+1-a\right) & =5 \zeta_{3}\left(2^{\alpha}\right) H(m)-3 S^{\prime}(m), \\
8 \sum F(4 a-3) \xi\left(\frac{m^{2}+3}{4}-a\right) & =H(m)-S(m), \\
8 \sum F(8 a-5) \xi\left(4 n^{2}+5-8 a\right) & =\left[7 \zeta_{3}\left(2^{\alpha}\right)+1\right] H(m), \\
\sum F(8 a-6) \xi\left(2 m^{2}+3-4 a\right) & =H(m), \\
8 \sum F(8 a-2) \xi\left(8 n^{2}+1-4 a\right) & =\left[7 \zeta_{3}\left(2^{\alpha+1}\right)+1\right] H(m) .
\end{aligned}
$$

Reversal of the order of summation and reduction of the right hand members give at once (I)-(VI), the pair (III), (IV) coming from (3.2).

UNIVERSITY OF WASHINGTON,

Seattle, Wash. 